Original Article

\title{
Homeopathy in the treatment of influenza: A data collection proposal
}

\author{
Flávio Dantas (MD DSc), Robert T. Mathie (MD), Joyce Frye (MD), \\ Chaturbhuja Nayak (MD)
}

On behalf of the International Scientific Committee on Homeopathy and Influenza (ISCHI)

\begin{abstract}
Influenza is a common disease, widespread all over the world, that has no satisfactory treatment up to now. Homeopathy has a long tradition of being used to treat influenza and has accumulated empirical and experimental evidence showing positive effects. Large scale observational studies in different continents have never been carried out to assess the overall benefits of homeopathy in flu, what prescription strategy and medicines are more effective and for which group of patients it can be most helpful. On the other hand, there is a predicted pandemic of avian flu and it is important to be proactive and to be prepared to test homeopathy in this context. This worldwide study will collect observational data on patients with influenza or influenza-like illness treated with homeopathic by experienced homeopathic doctors during a period of 2 years. Measurements will include duration of influenza illness, change in total score of symptoms and clinical scales, as well as safety aspects (presence of adverse effects and complications). Homeopathic and any conventional medicines prescribed will be registered. Data will be entered and loaded in a web application, and will be statistically analyzed using descriptive and inferential techniques. Ethical approval will be sought, and all cases are to be submitted in an anonymous way to protect patient identity. This project aims to enable collection of useful data, together with the development of information technology infrastructure, that could be useful in future multi-centre randomized controlled trials in the treatment of influenza patients with homeopathy, besides testing a new strategy for accumulating evidence on the benefit of homeopathic treatment in other diseases and conditions.
\end{abstract}

Keywords: Influenza; Homeopathy; Data collection; Evidence; Information technology database

\section{Introduction}

The evaluation of therapeutic technologies for medical use has been characterized by a tension between the implementation of strategies based on biomedical models - emphasizing efficacy, statistical significance, and group analysis - and the use of strategies based on biopsychosocial models that favor effectiveness, clinical relevance and the analysis of individual results [1]. Human beings are also both similar to other human beings (supporting the biomedical approach) and different from all other human beings (corroborating the biopsychosocial model).

Besides this, medicine and physicians face daily decisions that require certainty in uncertain circumstances, leading the great physician William Osler to state in 1904 that "Medicine is a science of uncertainty and an art of probability" [2].
According to the International Epidemiology Association, efficacy refers to the extent in which a specific intervention, procedure, regime or service produces a benefit under ideal conditions. On the other hand, effectiveness indicates the point in which they produce what is expected for a certain population when used under routine circumstances [3].

In the clinical area, randomized controlled trials (RCT) are regularly done to investigate the efficacy of interventions and observational studies for evaluating effectiveness. Evidence-Based Medicine (EBM) considers study design a decisive factor for judging the scientific validity of conclusions, giving the highest position to experimental designs (RCT) [4]. Observational (or non-experimental studies) are epidemiologic studies in which phenomena are allowed to occur according to their nature, without any intervention, with changes in one characteristic being studied in relation to change in other 
characteristics. Case-control studies and cohorts are examples of observational epidemiologic studies in which the researcher observes, without intervention and only statistically records, classifies, measures and analyzes the outcomes [3]. Outcome studies aim to assess the quality and effectiveness of health care by the accomplishment of a specified end result and are placed in a midpoint on the hierarchy of evidence according to EBM.

Homeopathy has been scientifically studies since its beginning, and there is room for more investigation and evaluation of its effects. It is particularly interesting to know the clinical significance of the homeopathic treatment in highly prevalent conditions and illnesses where there are effectiveness gaps, that is, clinical areas with unsatisfactory or no well-established treatments [5]. Human influenza is an acute febrile viral respiratory illness, widespread all over the world, which occurs annually in outbreaks of varying severity. RCTs have shown partial efficacy for antiviral medicines (oseltamivir and zanamivir) [6] and for homeopathic oscillococcinum [7], but there is no reliable collection of large data showing clear effects of traditional homeopathic medicine on the natural history of influenza (Dewey, [8] in 1921, published anecdotal observations of 50 American homeopathic doctors with very positive results on treating influenza).

Large scale clinical observational studies in different continents have never been carried out to assess the overall benefits of homeopathy in flu, what prescription strategy and medicines are most effective and for which group of patients it can be most helpful. On the other hand, there is a predicted pandemic of avian flu and it is important to be proactive and to be prepared to test homeopathy in this context.

\section{A model to asses homeopathy in a clinical setting}

The assessment of the effectiveness in homeopathy has been done in different settings and by using various research designs. In the $1990 \mathrm{~s}$, researchers at the Royal London Homoeopathic Hospital conducted series of outcomes systematic correlation for patients with rheumatoid arthritis and atopic dermatitis, [9,10] that went through consultation. Based on their experience, the authors recommended performing prospective studies, with protocols specifically designed for functional assessment and follow-up of patients. Evolving from this experience, a general proposal to assess the effectiveness of homeopathy in the treatment of common health problems in the realistic context of medical practice was published several years ago [11] and is the groundwork for the current project to collect data regarding influenza and homeopathic therapeutics. Conceptual foundations are:
1. Ethical orientation towards the well-being of patients and improving health care from a collective perspective

- Patients will use their autonomy to choose the treatment they consider most adequate for them (bioethical principle of autonomy);

- Physician's freedom to establish the therapeutic measures that he or she considers appropriate for the patient (principle of autonomy + principle of beneficence/non-maleficence);

- Preferential use of a model that maintains normal interaction between the physician and the patient, yet capable of improving it even more;

- Use in common or relevant problems - not life-threatening - usually treated exclusively or almost exclusively by homeopathic remedies with results empirically reported to be positive;

- Informed consent is unnecessary if the patient decides on his/her own will to seek a center/physician that offers homeopathic consultation. In other cases, the patient will be asked whether or not he/she accepts to be treated homeopathically (informed consent);

- Patient must be provided the best available treatment (use of homeopathy or whatever options are recommended in the given case).

2. Opening to different homeopathic treatment options based on the core concepts of homeopathy (principle of similarity + use of dilutes and agitated medicines, adapted to each patient)

- Respect for the specificity of homeopathic treatment and its global and individualized approach, as well as the different prescription patterns used by homeopathic doctors;

- Higher applicability as a consequence of the assessment of outcomes in clinical practice;

- General assessment of the patient (short/medium term $\square$ long term).

3. Simplicity, with increasing levels of complexity

- Focus only on essential information and procedures needed to comprehend/understand the main questions, including basic descriptions of patients and their prognostic factors;

- Broad - yet clearly defined - inclusion criteria in order to facilitate recruitment; 
- Minimum data collection, allowing inclusion of study procedures of routine clinical practice and their incorporation in the medical chart.

\section{Rigorous methodological design}

- Study must be prospective;

- Application of principles used in RCTs in order to improve the internal validity of the study, particularly validated or broadly accepted diagnostic criteria for the health problem, use of clinical measurement tools that have been validated, or that are usually employed in controlled therapeutic studies, degrees of severity of the problem, among others [12];

- Each patient manifests an illness in his/her particular way and with different levels of severity. Consequently, it is imperative to define beforehand the clinical spectrum of the disease, expressed in the terms of symptoms, morphology, associated comorbidity and other manifestations;

- Measure of the important effects of clinical treatment - effects recognized both by patients and physicians - after a previous statement of the expected minimum improvement [13];

- Measure of the perception of health and general functioning/quality of life factors as well as symptoms or specific measures (biological or functional) regarding the focused health problem in order to characterize the condition that is being treated and verifying the success of treatment [14];

- Use of data provided by the physician and patients regarding the evolution of the problem;

- Use of a broad sample, with pre-established minimum and maximum limits for participating physicians;

- Selection of a set of questions for each clinical problem in order to meet the data needed for each particular study;

- Use of clinimetric principles and indexes/semi-quantitative scores or ordinary scales or quasi-dimensional marks in a visual analog line [15] in order to assess human reactions that cannot be measured with any technological/laboratory tests;

- Identification of significant comorbidity [16] and prior treatments starting at the time of inclusion in the study.

\section{Interactivity and evolution}

- Adaptability to different contexts;

- Generation of hypothesis $\square$ Testing of hypotheses;

- Basis on the patients' and physicians' experiences;

- Progressively sophisticated designs resulting from the knowledge obtained from previous studies, allowing qualitative research to be conducted (using discourse analysis and other methods) or validation of constitutional types/essential issues on remedies;

- Medium- and long-term temporal perspective;

- Opening to the cooperation of other health professionals - without specific training in homeopathic prescription - for comparative efficacy studies.

\section{Technical and economical feasibility}

- Study of problems that do not demand costly technological resources or sophisticated laboratory tests for diagnosis and follow-up of the patient and without current fully satisfactory treatment;

- Use of simple models in the beginning, that may become more complex, allowing doctors with insufficient knowledge of scientific methods to become more educated in methodological matters;

- Motivation to involve a larger number of homeopathic physicians in the process of systematic observation of their patients, which may contribute to improve the quality of provided healthcare;

- Expectancy of poor official support and financial resources to fund homeopathic research in health-care centers around the world, in order to develop and assess the effectiveness of homeopathy $\square$ simplification of the protocol and motivation of the physician.

\section{Elements of a clinical protocol}

Each clinical protocol shall be structured taking into account the specific goals of each project, observing the following criteria: simplicity, feasibility and methodological quality. It may contain a minimum number of mandatory elements; others will be added according to availability and viability. Table 1 summarizes the aforementioned elements. 


\begin{tabular}{|c|c|c|}
\hline Area & Mandatory items & Accessory items \\
\hline $\begin{array}{l}\text { Clinical } \\
\text { diagnosis }\end{array}$ & $\begin{array}{l}\text { - Validated or conventionally } \\
\text { accepted diagnostic criteria }\end{array}$ & $\begin{array}{l}\text { - Selective inclusion of patients with levels of predefined } \\
\text { clinical severity } \\
\text { - Use of more sophisticated technological/laboratory } \\
\text { resources } \\
\text { - Clinical diagnosis established by another physician, } \\
\text { not involved in the treatment }\end{array}$ \\
\hline $\begin{array}{l}\text { Record of } \\
\text { clinical } \\
\text { information }\end{array}$ & $\begin{array}{l}\text { - Comorbidity } \\
\text { - Degree of severity } \\
\text { - Identification of essential } \\
\text { symptoms for prescription }\end{array}$ & $\begin{array}{l}\text { - Complete separation of processes for data collection } \\
\text { and clinical consultation, performed by different people }\end{array}$ \\
\hline Treatment & $\begin{array}{l}\text { - Definition of the minimum } \\
\text { period of time for patient follow-up } \\
\text { - Description of all treatments in } \\
\text { use and medical recommendations } \\
\text { - Record of occurrences after } \\
\text { prescription } \\
\text { - Establishment of maximum } \\
\text { number of homeopathic remedies } \\
\text { for each patient } \\
\text { - Description of adverse effects and } \\
\text { complications }\end{array}$ & $\begin{array}{l}\text { - Criteria for prescription of remedies (number of } \\
\text { remedies, number of symptoms for prescription, rules } \\
\text { for recruitment, etc.) to be applied in all patients } \\
\text { included } \\
\text { - Selective use of certain homeopathic remedies that are } \\
\text { more effective and with more reliable homeopathic } \\
\text { information } \\
\text { - Standardized instructions to guide progressive } \\
\text { reduction of conventional treatment } \\
\text { - Use of comparative group treated conventionally by } \\
\text { the homeopathic physician or other doctors, including } \\
\text { use of placebo as comparison }\end{array}$ \\
\hline $\begin{array}{l}\text { Evaluation of } \\
\text { therapeutic } \\
\text { results }\end{array}$ & $\begin{array}{l}\text { - Measurement of the most } \\
\text { relevant clinical effects } \\
\text { - Simple evaluation instruments } \\
\text { (scales, indexes), preferably } \\
\text { validated or conventionally } \\
\text { accepted }\end{array}$ & $\begin{array}{l}\text { - More intensive diagnostic and laboratory follow-up } \\
\text { - Evaluation of patients quality of life - generic, } \\
\text { disease-specific or individualized measures - or of well- } \\
\text { being in different periods of the study } \\
\text { - Observation period prior to treatment (waiting list) or } \\
\text { with use of conventional treatment or placebo }\end{array}$ \\
\hline $\begin{array}{l}\text { Evaluation of } \\
\text { patient's } \\
\text { economic } \\
\text { status and } \\
\text { satisfaction }\end{array}$ & $\begin{array}{l}\text { - Satisfaction degree with } \\
\text { treatment } \\
\text { - Measurement of time spent by } \\
\text { the physician }\end{array}$ & $\begin{array}{l}\text { Cost-effective analysis, including absenteeism, } \\
\text { expenses with transportation, lost working hours, } \\
\text { consultation with other physicians, etc }\end{array}$ \\
\hline
\end{tabular}

\section{A Proposal for Data Collection in Homeopathy and Influenza}

In the context of a predicted pandemic of avian flu, an ongoing international data collection study of seasonal flu would enable a greater understanding of its homeopathic management and would establish the infrastructure to help to identify and study the genus epidemicus that may be relevant in avian flu, as well as facilitate more rigorous clinical studies on homeopathy and influenza. It will help to facilitate the creation of a research culture among homeopathic medical practitioners worldwide making, thus, possible to establish in the future a worldwide network for clinical studies in different medical conditions using homeopathic medicines.

Homeopathy is widely used in the treatment of influenza, but there is no systematic collection of clinical data in different centers distributed worldwide. A systematic analysis of the use of different approaches and homeopathic medicines by experienced doctors will help to suggest better prescription strategies and the most useful homeopathic medicines for treating human influenza.

This project will enable the collection of data together with information technology infrastructure that may be useful to implement future multi-center RCTs in the treatment of influenza patients with homeopathy.

A large effort in collecting data on the homeopathic treatment of flu is supported by different reasons, including:

- the need to apply modern scientific methods to the evaluation of homeopathy (evidencebased medicine); 
- the difficulty to apply results from RCTs to the "real world";

- the value of observational studies in the appraisal of clinical practice and informing worthwhile research, particularly when carried out in large numbers of patients;

- the impact of suggesting effective results on treating common and epidemic acute conditions using homeopathic medicines;

- the need to develop the knowledge of symptoms and genus epidemicus of influenza and its management by homeopathic medical practitioners;

- the relevance of creating a network of homeopathic practitioners worldwide to gather data on homeopathic treatment in influenza;

- the application of modern information technology tools and techniques to collect and analyze data from clinical practice.

The project aims are:

- to build a database and web application to be used in the assessment of influenza treated with homeopathic medicines

- to recruit and establish a network of motivated homeopathic physicians to collaborate in data collection and, in the future, in clinical trials

- to assess case report forms completion by providers, including full symptom documentation and follow-up

- to identify the pattern of symptoms most used for homeopathic prescription in clinical cases of influenza/avian flu diagnosed according to predefined criteria (confirmed or not by virological diagnosis)

- to evaluate homeopathy (alone or in combination with other treatments) in the treatment of influenza/avian flu in large numbers of patients

- to assess different homeopathic strategies of prescription in treating influenza

A large number of patients with all types of influenza or influenza-like illness (laboratory confirmed influenza or clinically defined influenza) will be included, after attention in the usual clinical practice of homeopathic doctors (outpatient departments, private offices, hospitals). Only officially licensed homeopathic doctors in each country, with at least 3-year experience in prescribing homeopathic medicines and previous experience in homeopathically treating patients with flu will be recruited to take part in the study. Centers will be established in countries with large population, traditional use of homeopathy by people and significant number of well trained homeopathic doctors.

A pilot study, with the inclusion of 500 patients is to be carried out in 2008-2009. At the end of the project it is expected to identify the most common symptoms presented by patients with influenza in different parts of the world (genus epidemicus) and the description of the seemingly most effective homeopathic medicines and prescription strategies for influenza. Among other candidates, centers may be created in Belgium, Brazil, Cuba, France, Germany, India, Italy, Mexico, Netherlands, Spain, United Kingdom, United States of America and countries in Eastern Europe and Asia.

A case report form (CRF) was developed for entering data at the first and follow-up consultations. Entry criteria were defined after survey of the literature on human influenza and recent RCTs published in the main clinical journals.

To include a patient, criteria are: 1) reports of influenza virus in the community; 2) acute onset (less than 36 hours); 3) body temperature at least $37.7 \mathrm{oC} / 99.8 \mathrm{oF}$ and 4 ) at least one among: fatigue, malaise, sweats/chills, myalgia, headache and one among: cough, rhinorrhea, sore throat, nasal obstruction and red or tearful eyes. A global score will be calculated for each patient based on the intensity of the symptoms. Confidence in prescription for each medicine will be marked in a 4point scale. Homeopathic and any conventional medicines prescribed will be recorded. Medicines will be prepared by local homeopathic pharmacies or laboratories, as usual in the context of the practitioners.

Primary outcomes will include: 1) duration of the symptoms of influenza/time to resolution; 2) change in the total score of symptoms; 3) days lost from work/school/time to return to normal activity or school; 4) number and severity of adverse effects. Assessment of secondary outcomes measures for treatment will include: 1) time to reduction in severity of clinically significant symptoms; 2) use of other non-homeopathic treatments; 3) improvement/deterioration in clinical scales and 5) incidence of complications. Practitioners will evaluate patients using a specific form, either in person or over the telephone, according to their customary way to follow up patients in acute conditions.

Observational, non-randomized studies are better designed to do exploratory data analysis [17], additionally, data description strategies should be performed comprehensively, through graphs and 
charts or applying influence analysis or sensitivity analysis [18], according to Greenland's recommendations [19].

From an exploratory perspective (hypotheses generation), preliminary links between treatment received by patients, verified therapeutic outcomes and degree of confidence in the prescription must be analyzed, according to the different levels: levels of severity, differences in compliance to prescribed treatment, course of significant comorbidities and simultaneous use of additional treatments.

Data on the homeopathic treatment of influenza collected by officially licensed homeopathic doctors in usual practice will be uploaded to a central database. There will be no specific recruitment for this study: patients seeking homeopathic treatment for their condition will be treated as usual by their doctors, with periodic follow-up. Data of such patients with influenza-like symptoms will be entered by each doctor in a website and will be scrutinized for inclusion by a regional coordinator in each country or region. After inclusion, all patients are to be registered in the database and their results (or withdrawal) informed. Accepted data will then be checked and corrected as required before being submitted to a Central (International) Coordination.

The central database will be developed using an open source relational database management system (RDBMS). It will be accessed through a webbased dynamic site designed to offer information about the project, to upload data to the central database and to support the analysis and interpretation of data by the homeopathic doctors and researchers involved in the project.

Data collection will be supported by online completion of forms. Software will be designed to allow data entry in a user-friendly, safe and searchable way (respecting different levels of responsibility), particularly by using pick-lists for homeopathic medicines, common symptoms and modalities. An OLAP (online analytical processing) web application integrated to a data mart (a multidimensional database specially developed to support analysis and decision-making) will be developed to allow doctors to conduct flexible data analysis, exploring the data and establishing correlations between treatments and outcomes, particularly in homeopathic medicines prescribed with high confidence.

The website will make data from the central data mart available to practitioners in order to conduct their own ad hoc analyses (i.e. of their own data) and also will offer access to predefined analysis reports periodically executed centrally on the data mart.
Alternatively, doctors will be able to download subsets of data to their machine to be explored locally using suitable statistical software (such as SPSS or similar). These central and local facilities will support flexible data analysis and exploration, mainly the establishment of correlations between treatments and outcomes, particularly in homeopathic medicines prescribed with confidence. Data will be described and analyzed centrally by using suitable statistical software (such as SPSS), with correlations between treatments and outcomes, especially in homeopathic medicines prescribed with high confidence.

All cases are to be submitted in anonymous way, so that the identification of patients would only be possible to the attending physician. Homeopathic doctors will adopt their usual practice in treating patients with influenza, the only difference consisting in the application of a structured form in evaluation. Given the different national ethical regulations on research and on the legal use of homeopathy worldwide, requirements for approval by ethics committees will be considered in a country-by-country basis.

\section{International Scientific Committee on Homeopathy and Influenza (ISCHI):}

Peter Fisher (UK, Chair), Chaturbhuja Nayak (Índia), Flávio Dantas (Brasil), Gilles Chauffern (France), Helmut Roniger (UK), Jennifer Jacobs (USA), John Ives (USA), Joyce Frye (USA), MarieFrance Bordet (France), Menachen Oberbaum (Israel), Michel van Wassenhoven (Belgium), Robert Mathie (UK).

\section{References}

[1] Diamond GA, Denton TA. Alternative perspectives on the biased foundations of medical technology assessment. Ann Intern Med. 1993; 118: 455-464.

[2] Osler W, Silverman ME, Murray TJ, Bryan CS. The quotable Osler. Philadelphia: ACP Press; 2003.

[3] Last JM, editor. A dictionary of epidemiology. $3^{\text {rd }}$ ed. New York: Oxford University Press; 1995.

[4] Guyatt GH, Haynes RB, Jaeschke RZ, Cook DJ, Green L, Naylor CD, et al. For the Evidence-Based Medicine Group: Users' guides to the medical literature. XXV: Evidence-Based Medicine: principles for applying the users' guides to patient care. JAMA. 2000; 284: 1290-1296.

[5] Fisher P, van Haselen R, Hardy K, Berkovitz S, McCarney R. Effectiveness gaps: a new concept for evaluating health service and research need applied to complementary and alternative medicine. J Altern Complement Med. 2004; 10(4): 627-632. 
[6] Jefferson T, Demicheli V, Rivetti D, Jones M, Di Pietrantonj C, Rivetti A. Antivirals for influenza in healthy adults: systematic review. Lancet. 2006; 367(9507): 303-313.

[7] Vickers AJ, Smith C. Homoeopathic Oscillococcinum for preventing and treating influenza and influenza-like syndromes. Cochrane Database Syst Rev. 2006 Jul 19; 3: CD001957.

[8] Dewey WA. Homeopathy in influenza: a chorus of fifty in harmony. J Am Inst Homeopathy. 1921; 11: 1038-1043.

[9] Van Haselen R, Fisher P. Towards a new method for improving clinical homeopathy. Br Hom J. 1992; 81: $120-126$.

[10] Fisher P, Sharples F, Dantas F. Royal London Homoeopathic Hospital 500 cases analysis. Proceedings of IV Symposium of Boiron Institute: Rencontres Homéopathie \& Dermatologie; 2000 Mar, Apr 31-01; Paris, France. Paris: Boiron; 2000. 49-58.

[11] Dantas F. Avaliação de resultados terapêuticos da homeopatia: uma proposta para a realidade brasileira. Revista de Homeopatia. 2003; 68(1): $47-$ 62.

[12] Horwitz RI, Feinstein AR. The application of therapeutic-trial principles to improve the design of epidemiologic research: a case-control study suggesting that anticoagulants reduce mortality in patients with myocardial infarctation. J Chron Dis. 1981; 34: 575-583.

[13] Naylor CD, Llewelyn-Thomas HA. Can there be a more patient-centered approach to determining clinically important effect sizes for randomized treatment trials? J Clin Epidemiol. 1994; 47: 787795 .

[14] Wilson IB, Cleary PD. Linking clinical variables with health-related quality of life: a conceptual model of patient outcomes. JAMA. 1995; 273: 59-65.

[15] Feinstein AR. Clinical judgment revisited: the distraction of quantitative models. Ann Intern Med. 1994; 120: 799-805.

[16] Katz JN, Chang LC, Sangha O, Fossel AH, Bates DW. Can comorbidity be measured by questionnaire rather than medical record review? Med Care. 1996; 34: 73-84.

[17] Tukey JW. Exploratory data analysis. Reading: Addison-Wesley; 1977.

[18] Rosenbaum PR. Discussing hidden bias in observational studies. Ann Intern Med. 1991; 115: 901-905.

[19] Greenland S. Randomization, statistics and causal inference. Epidemiology 1990; 1: 421-429.

(c)) BY-NC-ND Licensed to GIRI

Support: authors declare that this study received no funding

Conflict of interest: authors declare there is no conflict of interest

Revised 15 June 2008; Published: 30 June 2008

Revised version of presentation at 63rd Congress of LMHI, Oostende, May 20-24th, 2008

Erratum: 30 Dec 2008. (http://www.feg.unesp.br/ ojs/zacha_ijhdr/erratum/?v=7\&i=23\&pi=56)

Correspondence author: Flavio Dantas, dantasfla@gmail.com

How to cite this paper: Dantas F, Mathie RT, Frye J, Nayak C. Homeopathy in treatment of influenza: A data collection proposal. Int J High Dilution Res [online]. 2008 [cited YYYY Mmm DD]; 7(23): 56-62. Available from: http://www.feg.unesp.br/ ojs/index.php/ijhdr/article/view/284/352. 